\title{
HER-2 Signaling, Acquisition of Growth Factor Independence, and Regulation of Biological Networks Associated with Cell Transformation
}

\author{
Aliccia Bollig-Fischer, Michele Dziubinski, Alaina Boyer, Ramsi Haddad, Craig N. Giroux, \\ and Stephen P. Ethier \\ Breast Cancer Biology Program, Karmanos Cancer Institute, Wayne State University School of \\ Medicine, Detroit, Michigan
}

\begin{abstract}
Activated oncogenes are the dominant drivers of malignant progression in human cancer, yet little is known about how the transformation from proto-oncogene to activated oncogene drives the expression of transformed phenotypes. An isogenic model of HER-2-mediated transformation of human mammary epithelial cells was used along with HER-2-amplified human breast cancers to investigate how HER-2 activation alters its properties as a signaling molecule and changes the networks of HER-2-regulated genes. Our results show that full oncogenic activation of HER-2 is the result of a transition in which activated HER-2 acquires dominant signaling properties that qualitatively alter the network of genes regulated by the activated oncogene compared with the proto-oncogene. Consequently, gene expression programs related to invasion, cell stress, and stemness become regulated by HER-2 in a manner not observed in nontransformed cells, even when HER-2 is overexpressed. Our results offer novel insights into biological processes that come under the control of HER-2 after it acquires full oncogenic potential.
\end{abstract}

\section{Introduction}

HER-2 is one of the most well studied of all human cancer genes. HER-2 is a causal factor in $\sim 25 \%$ of breast cancers and has a role in development of other cancer types as well (1-3). HER-2 has dominant transforming activity in vitro, and targeted overexpression of HER-2 in transgenic mice causes development of mammary tumors (4-10). As a signaling molecule, HER-2 is a type I receptor tyrosine kinase with no natural ligand, but with the ability to interact with other HER family members following binding of their cognate ligands $(11,12)$. In addition, there is substantial evidence that HER-2 can form constitutively active homodimers that signal in a ligand-independent manner when the receptor is overexpressed in cancer cells $(13,14)$. Despite the wealth of knowledge about the HER-2 oncogene, many questions remain unanswered about the biological basis for the transformed phenotypes induced by $H E R-2$ when it is amplified and overexpressed.

Previously, we showed that unlike normal breast epithelial cells, breast cancer cells with amplification and overexpression of HER-2 exhibit independence of both insulin- and

(C) 2010 American Association for Cancer Research.

Corresponding Author: Stephen P. Ethier, Breast Cancer Biology Program, Karmanos Cancer Institute, Wayne State University School of Medicine, 4100 John R Street, HWCRC, Room 840.1, Detroit, MI 48201. Fax: 313-576-8626; ethier@karmanos.org.

Disclosure of Potential Conflicts of Interest

No potential conflicts of interest were disclosed.

Note: Supplementary data for this article are available at Cancer Research Online (http://cancerres.aacrjournals.org/). 
epidermal growth factor (EGF)-like growth factors for continuous proliferation under serum-free conditions (15-20). We then showed that the transition to growth factor independence can be mimicked by overexpression of HER-2 in MCF-10A cells, which results in insulin independence, and by coexpression of HER-2 and human papillomavirus (HPV)-E7, which results in insulin- and EGF-independent growth. The multiple growth factor independence induced by these cooperating oncogenes is identical to what we observed in HER-2-amplified breast cancer cells (6, 20, 21). Furthermore, EGF-independent MCF-10HER2/E7 cells are highly tumorigenic in vivo and can invade and form bone metastases. The HER-2-amplified and EGF-independent SUM-225 and SUM-190 cells are also tumorigenic in vivo (22). In contrast, EGF-dependent MCF-10HER2 cells are partially transformed in that they form colonies in soft agar, but are not tumorigenic in vivo. Thus, the transition to complete growth factor independence is a hallmark of the full transforming function of the HER-2 oncogene.

In the present studies, we sought to explore how signaling from the HER-2 oncoprotein changes as HER-2 acquires full transforming activity and as cells undergo the transition to complete growth factor independence. We also investigated the downstream consequences of HER-2 signaling in growth factor-dependent and growth factor-independent cells to connect changes in gene expression with the acquisition of biological properties associated with fully transformed cells. The results of these experiments show that for EGF-dependent but HER-2-overexpressing cells, ligand-mediated EGF receptor (EGFR) activation is the driver of HER-2 tyrosine phosphorylation, which results in significant alterations in downstream gene expression compared with cells expressing physiologic levels of HER-2. More importantly, however, in HER-2-overexpressing but fully growth factor-independent cells, HER-2 is the driver of EGFR and HER-3 tyrosine phosphorylation, which results in still further alterations in gene expression that are linked to altered biological properties of transformed cells. These results shed new light on the mechanisms by which the fully activated HER-2 oncogene induces malignant transformation.

\section{Materials and Methods}

\section{Cell lines, cell culture conditions, nuclear extracts, whole-cell lysates, and cell proliferation assays}

Details on these methods are presented in Supplementary Materials and Methods.

\section{Immunoprecipitation and immunoblotting}

Immunoprecipitation and Western blotting were performed as previously described (21). Cells were lysed in a buffer containing $20 \mathrm{mmol} / \mathrm{L}$ Tris- $\mathrm{HCl}(\mathrm{pH} 8.0), 137 \mathrm{mmol} / \mathrm{L} \mathrm{NaCl}$, $1 \%$ NP40, $10 \%$ glycerol, $1 \mathrm{mmol} / \mathrm{L} \mathrm{Na}_{3} \mathrm{VO}_{4}, 1 \mathrm{mmol} / \mathrm{L}$ phenylmethylsulfonyl fluoride, $1 \%$ aprotinin, and $20 \mu \mathrm{g} / \mathrm{mL}$ leupeptin. Protein concentrations were equalized using the Bradford method. For whole-cell lysates, Laemmli sample buffer was added and the samples were boiled. For immunoprecipitation, $1 \mu \mathrm{g}$ of antibody was added to $1 \mathrm{mg}$ of sample and incubated at $4^{\circ} \mathrm{C}$ for 1 hour. Immune complexes were then bound to protein $\mathrm{A} / \mathrm{G}$ beads for 1 hour at $4^{\circ} \mathrm{C}$. Immunoprecipitates were washed three times in lysis buffer. Laemmli sample buffer was added and the samples were boiled. EGFR immunoprecipitates were loaded onto 7.5\% SDS-polyacrylamide gels. After proteins were bound to polyvinylidene difluoride membranes by semidry electrophoretic transfer with a Bio-Rad Transblot SD unit, membranes were probed with primary antibodies following the supplier's recommendation and secondary peroxidase-conjugated antibodies (anti-mouse or anti-rabbit) from Vector Laboratories. Immunoreactive protein was visualized by enhanced chemiluminescence with reagents from Pierce Laboratories/Thermo Scientific. Required antibodies are as follows: anti-EGFR (MAB108; gift from Julie Boerner, Karmanos Cancer Institute), anti-HER-2 
(Ab-17; Lab Vision), anti-EGFR (Zymed Laboratories), phosphotyrosine (BIOMOL), antiHER-3 (Ab-2; Lab Vision), and anti-HER-3 (C-17; Santa Cruz Biotechnology). Antibodies for SHC, eIF4E, GAB-1, 4E-BP1, extracellular signal-regulated kinase 1/2 (ERK1/2), AKT, pS6, and eIF45 are from Cell Signaling Technology. For hypoxia-inducible factor-1a (HIF-1a) immunoblots, a similar procedure was followed, except that cells were treated with $\mathrm{CoCl}_{2}(150 \mu \mathrm{mol} / \mathrm{L})$ for 16 hours to stabilize HIF-1a protein before nuclear extraction. Anti-HIF-1a antibody used at 1:1,000 dilution, raised in goat, was from R\&D Systems. Secondary peroxidase-labeled anti-goat at 1:2,500 dilution was from Vector Laboratories. Gels were imaged by a Molecular Imager and Universal Hood II, and Quantity One software from Bio-Rad Laboratories.

\section{Semiquantitative reverse transcription-PCR}

RNA was extracted from cells using the Qiagen RNeasy kit. RNA was converted into cDNA via a reverse transcription reaction using oligo(DT) primers and the SuperScript III FirstStrand Synthesis System (Invitrogen). Primer sets specific to $~ 100-b p$ sequences of target genes and controls (PUM1 and GAPDH) were ordered from Invitrogen. Quantitative reverse transcription-PCR (RT-PCR) was done in $25 \mu \mathrm{L}$ reactions, in 96-well plates, using 100 or 200 ng cDNA and the FastStart SYBR Green Master Mix (Roche Diagnostics). Reactions were done at minimum twice in duplicate using the Bio-Rad iQ5 real-time PCR machine (Bio-Rad Laboratories). Cycles to threshold values were normalized to values for GAPDH and PUM1 and calibrated to MCF-10A cell levels. Control wells containing PCR master mix and primers without sample cDNA emitted no fluorescence after 40 cycles. Relative expression data were calculated as described by Livak and Schmittgen (23).

\section{Microarrays and gene expression analysis}

Gene expression networks from MCF-10A, MCF-10HER2, and MCF-10HER2/E7 cells were determined from an analysis of global gene expression time series data. Cells were plated so that they reached $75 \%$ confluency after 4 days. At this point, cultures were treated with the EGFR kinase inhibitor gefitinib $(0.5 \mu \mathrm{mol} / \mathrm{L})$ or HER-2 kinase inhibitor CP724,714 $(1 \mu \mathrm{mol} / \mathrm{L})$ and RNA was isolated from parallel plated plates at $0,3,6,9,12,15,18,21,24$, $27,30,33,36,39,42$, and 45 hours after addition of inhibitor. Medium was changed the day after plating and at the start of treatment. Quantity measurement and the high quality of all mRNA samples were assured by analysis with the NanoDrop 1000 Agilent Bioanalyzer and the Agilent RNA 6000 Nano kit (Agilent Technologies). Expression levels at each time point for each cell and treatment were determined by microarray analyses using the Illumina human Ref8v2 array. Microarray analyses were performed by the Genomics Core of the Karmanos Cancer Institute. Data were processed for quality control and normalized across compared arrays by quantile normalization. Two thousand and five hundred genes with 1.7 or greater expression fold change at any time point in the series were included in Ingenuity Pathway Analysis. Cluster analysis of expression profiles was performed with Bayesian analysis using CAGED (cluster analysis of gene expression dynamics) software. A detailed description of the application of this method and the statistical goodness of fit for each cluster are in the Supplementary CAGED Report. Canonical pathway analysis identified the pathways from the Ingenuity Pathway Analysis library of canonical pathways that were most significant to the data set. Molecules from the data set that met the 1.7-fold change cutoff and were associated with a canonical pathway in Ingenuity's Knowledge Base were considered for the analysis. The significance of the association between the data set and the canonical pathway was measured in two ways: $(a)$ a ratio of the number of molecules from the data set that map to the pathway divided by the total number of molecules that map to the canonical pathway and $(b)$ Fisher's exact test was used to calculate a $P$ value determining the probability that the association between the genes in the data set and the canonical pathway is explained by chance alone. 


\section{Measurement of reactive oxygen species}

Reactive oxygen species (ROS) levels were measured in live cells using the Image-iT LIVE Green Reactive Oxygen Species Detection kit (Molecular Probes, Inc.). Cells were exposed to a fluorescein compound that when oxidized emits green fluorescence that was observed by fluorescence microscopy using a Nikon Eclipse TE2000-U microscope (excitation, 495 $\mathrm{nm}$; emission, $529 \mathrm{~nm}$ ). In addition, nuclei were stained with Hoechst 3342 (excitation, 350 $\mathrm{nm}$; emission, $461 \mathrm{~nm}$ ).

\section{Reporter assays}

HIF-1a reporter assays were performed in MCF-10A, MCF-10HER2, and MCF-10HER2/ E7 cells using Cignal Reporter Assay kits (SABiosciences). Lipofectamine transfection reagent (Invitrogen) was used to transfect the reporter constructs (tandem repeats of the HIF-1a element with minimal cytomegalovirus promoter) and negative (minimal promoter only) and positive [green fluorescent protein (GFP)-expressing construct] controls into $2 \times$ $10^{4}$ cells in 96-well clear-bottom plates. After 12 to 16 hours of transfection, the cells were changed into complete growth medium and incubated at $37^{\circ} \mathrm{C}, 10 \% \mathrm{CO}_{2}$, at either $5 \% \mathrm{O}_{2}$ or atmospheric $\mathrm{O}_{2}$. After 2 days, luciferase activity was determined using the dual luciferase assay system (Promega), measured using the Victor3 1420 multilabel counter (PerkinElmer). The relative reporter activity values were expressed as arbitrary units per $\mu \mathrm{g}$ protein measured using the Bradford assay protocol. Transfection efficiency was determined by monitoring GFP expression in transfected cells by fluorescence microscopy using a Nikon Eclipse TE2000-U microscope. The entire experiment was repeated, in triplicate, and the SD was calculated for each experiment.

\section{Results}

To examine the changes in HER-2 signaling that occur as HER-2-overexpressing cells undergo the transition to growth factor independence, we took advantage of a panel of wellcharacterized cell lines developed in our laboratory, as well as specific inhibitors of either HER-2 or EGFR. The cell lines used include the isogenic cell line series MCF-10A, MCF-10HER2 (EGF-dependent), and MCF-10HER2/E7 (EGF-independent) and the HER-2-amplified human breast cancer cell lines SUM-225, SUM-190, 21MT1 (EGFindependent), and 21MT2 (EGF-dependent). We performed experiments with CP724,714, a HER-2-specific small-molecule kinase inhibitor that was recently described in detail (24). We also used the EGFR-selective kinase inhibitor gefitinib at concentrations that are selective for EGFR. The HER-2 and EGFR expression characteristics of the cell lines used in these experiments, as well as data on the specificity of CP724,714 for HER-2 activity, are shown in Supplementary Fig. S1. These data show that HER-2 expression levels in MCF-10HER2 and MCF-10HER2/E7 cells are 10- to 15-fold higher than parental MCF-10A cells, and HER-2-amplified breast cancer cell lines express HER-2 protein levels that are 1.5- to 2-fold higher than HER-2-overexpressing MCF-10A cells. SUM-225 and MCF-10A cells express similar levels of EGFR, and SUM-190 cells, despite having HER-2 amplification, do not express detectable levels of EGFR protein.

Next, we examined the effect of CP724,714 on EGFR tyrosine phosphorylation in the isogenic MCF-10 cell line series and in SUM-225 cells. As expected, exposure of MCF-10A cells or the EGF-dependent MCF-10HER2 cells to the HER-2-specific drug CP724,714 had no effect on tyrosine phosphorylation of EGFR (Fig. 1A; Supplementary Fig. S1C). By contrast, tyrosine phosphorylation of EGFR was completely inhibited by CP724,714 in the EGF-independent MCF-10HER2/E7 and SUM-225 cells (Fig. 1A). In addition, immunoprecipitation of EGFR from MCF-10HER2/E7 and SUM-225 cells resulted in coimmunoprecipitation of HER-2, and tyrosine phosphorylation of both EGFR and HER-2 
was inhibited by CP724,714, an effect not observed in the EGF-dependent cells. The changes detected in these cell lines following exposure to CP724,714 were observable within 5 minutes of treatment with drug (Fig. 1B). Next, we repeated the experiment but with the addition of an experimental group consisting of MCF-10HER2/E7 cells that had been exposed to EGF overnight. The results of this experiment once again showed inhibition of EGFR and HER-2 tyrosine phosphorylation by CP724,714 in EGF-independent MCF-10HER2/E7 and SUM-225 cells, but not in MCF-10HER2 cells (Fig. 1C). However, CP724,714 no longer blocked EGFR tyrosine phosphorylation when MCF-10HER2/E7 cells were stimulated with exogenous EGF. Thus, exposure of EGF-independent cells to EGF resulted in reversion to the signaling observed in EGF-dependent cells.

Next, we examined the effect of CP724,714 on proliferation of HER-2-overexpressing MCF-10A cells and human breast cancer cells. Figure 1D shows that whereas CP724,714 had no effect on growth of control MCF-10A cells, the drug slowed proliferation of MCF-10HER2 cells and completely inhibited growth of MCF-10HER2/E7 cells. Exposure to CP724,714 also completely inhibited proliferation of EGF-independent SUM-225, SUM-190, and 21MT1 cells (Fig. 1D, right). Thus, as HER-2-overexpressing cells progress to complete growth factor independence, they become increasingly reliant on HER-2 tyrosine kinase activity for signaling and proliferation.

We next performed the reciprocal experiments and examined the influence of EGFR tyrosine kinase activity on EGFR and HER-2 tyrosine phosphorylation in the panel of cell lines following exposure to gefitinib. Figure 2A shows that in the EGF-dependent MCF-10HER2 cell line, inhibition of EGFR kinase activity by exposure to gefitinib resulted in loss of EGFR tyrosine phosphorylation and a reduction in the level of HER-2 tyrosine phosphorylation. By contrast, exposure of EGF-independent MCF-10HER2/E7, SUM-190, or SUM-225 cells to gefitinib resulted in dramatically reduced levels of tyrosine-

phosphorylated EGFR, but had no effect on the level of HER-2 tyrosine phosphorylation. Furthermore, removal of EGF from the culture medium of MCF-10HER2 cells resulted in loss of tyrosine phosphorylation of both EGFR and HER-2, and this is not observed in EGFindependent cells (Fig. 2B).

These results indicate that in MCF-10A cells and in EGF-dependent MCF-10HER2 cells, EGF-stimulated EGFR tyrosine kinase activity is the driver of signaling and mediates both EGFR and HER-2 tyrosine phosphorylation. However, when cells undergo the transition to EGF independence, constitutive HER-2 tyrosine kinase activity is the driver of its own tyrosine phosphorylation and becomes the driver of EGFR tyrosine phosphorylation and cell proliferation.

Next, we measured the influence CP724,714 on HER-2 and EGFR tyrosine phosphorylation in four additional HER-2-amplified cell lines: 21MT1, 21MT2, BT474, and SKBR3. The $21 \mathrm{MT} 1$ cells are completely growth factor independent, but 21MT2 cells maintain their dependency on EGF $(19,25)$. Supplementary Fig. S2 shows that these breast cancer cell lines responded identically to CP724,714 treatment as the isogenic MCF-10A series, SUM-225 and SUM-190 cells.

We then examined the influence of HER-2 or EGFR kinase activity on the expression and tyrosine phosphorylation of HER-3. Figure 3A shows that three EGF-independent breast cancer cell lines express higher levels of HER-3 than the EGF-dependent 21MT2 cell line. In addition, whereas tyrosine-phosphorylated HER-3 was undetectable in the $21 \mathrm{MT} 2$ cells, it was readily detectable in the other three cell lines. Furthermore, HER-3 tyrosine phosphorylation was inhibited by CP724,714, but not by gefitinib in each of the EGFindependent cell lines, whereas gefitinib had no effect on HER-3 tyrosine phosphorylation in 
these cells. In the MCF-10 cell line series, overall expression of HER-3 was highest in the MCF-10HER2 cells compared with the other two cell lines (Fig. 3B). HER-3 tyrosine phosphorylation was low in MCF-10HER2 cells and, more significantly, was unaffected by gefitinib. Despite the relatively low levels of HER-3 expression and tyrosine phosphorylation observed in the MCF-10HER2/E7 cells, HER-3 tyrosine phosphorylation was inhibited by CP724,714 and not by gefitinib (Fig. 3C). Thus, in growth factorindependent cells, the HER-2 kinase was responsible for tyrosine phosphorylation of HER-3 as well as EGFR.

We then extended the analysis to downstream signaling molecules in two EGF-independent cell lines and one EGF-dependent breast cancer cell line. Supplementary Fig. S3 shows that in 21MT1 and SUM-190 cells, inhibition of HER-2 kinase activity, but not inhibition of EGFR kinase activity, resulted in loss of phosphorylation of ERK, AKT, SHC, and GAB-1. By contrast, in the EGF-dependent 21MT2 cells, phosphorylation of these downstream signaling molecules was inhibited by gefitinib and not by CP724,714.

In summary, our results show that the transforming activity of the fully activated $H E R-2$ oncogene, as indicated by expression of complete growth factor independence and tumorigenicity in vivo, is mediated by constitutive HER-2 kinase activity that is the dominant driver of signaling. As such, HER-2 kinase activity mediates tyrosine phosphorylation of EGFR, HER-3, and downstream signaling molecules. This is in contrast to EGF-dependent cells, even those that overexpress HER-2, in which ligand-mediated EGFR activation is the driver of HER-2 tyrosine phosphorylation and downstream signaling.

\section{Regulation of gene expression by HER-2 in HER-2-overexpressing cells}

To examine how HER-2-mediated signaling influences the expression of transformed phenotypes, we measured the changes in gene expression following exposure of these cell lines to EGFR or HER-2 kinase inhibitors. MCF-10A, MCF-10HER2, and MCF-10HER2/ E7 cells were exposed to either CP724,714 or gefitinib, and RNA was isolated for wholegenome expression profiling every 3 hours over a 48 -hour period. We chose a time series approach because single time point gene expression profiling studies only provide a snapshot in time of the state of a cell population and provide a limited description of the complexity of the signaling response. In contrast, multiple time point experiments provide richer data sets that capture the dynamics of the response of the system, which is important for further mechanistic studies. Time series experiments also allowed us to use robust computational tools such as CAGED (26) and Ingenuity Pathway Analysis to shed light on the novel biological properties regulated by HER-2 in the context of various conditions of transformation. For this analysis, we included 2,500 of the most dynamic genes, which exhibited a 1.7-fold or greater change in expression level at any time point during the experiment. As part of the time series experiments, we confirmed that MCF-10A and MCF-10HER2 cell proliferation was significantly attenuated within 48 hours of treatment with $0.5 \mu \mathrm{mol} / \mathrm{L}$ gefitinib, and that MCF-10HER2/E7 cell proliferation was significantly attenuated by treatment with $1 \mu \mathrm{mol} / \mathrm{L}$ CP724,714 (Supplementary Fig. S4).

We used the Bayesian model-based clustering method CAGED (26) to analyze the time series expression of three groups of genes to identify sets of coregulated genes in each cell line. The gene groups analyzed included ( $a$ ) genes commonly regulated by EGFR/HER-2 signaling in all three cell lines; $(b)$ genes regulated by EGFR/HER-2 signaling in both MCF-10HER2 and MCF-10HER2/E7 cells, but not in MCF-10A cells; and (c) genes regulated by HER-2/EGFR signaling only in the fully transformed MCF-10HER2/E7 cells, but not regulated by EGFR/HER-2 signaling in the other two cell lines. The CAGED method identified clusters of genes for each group that displayed similar temporal patterns of expression following exposure to kinase inhibitors (Supplementary CAGED Report). We 
found that 414 genes were commonly regulated by EGFR/HER-2 signaling in all three cell lines. The expression of 224 genes was positively regulated by EGFR/HER-2 signaling as indicated by their expression in nontreated cells and the progressive decrease in expression over time following drug treatment. In contrast, expression of 190 genes was negatively regulated by EGFR/HER-2 signaling in the three cell lines. CAGED analysis of these 414 genes resulted in the identification of 33 distinct gene expression clusters (Supplementary CAGED Report, pages 1-3). Complete time series data for two genes commonly regulated in the three cell lines are shown in the top two panels of Fig. 4. We next sought to identify genes that were regulated by EGFR/HER-2 signaling in the two HER-2-overexpressing lines, but not by EGFR in MCF-10A cells. This analysis resulted in the identification of 212 genes that were altered in their expression by exposure of MCF-10HER2 and MCF-10HER2/E7 cells to either gefitinib or CP724,714. Of these, 108 genes were positively regulated, and 104 genes were negatively regulated by EGFR/HER-2 signaling. CAGED analysis of the expression profiles of this 212-gene set resulted in the identification of 13 clusters (Supplementary CAGED Report, pages 36-37). Complete time series data for two genes from this group are shown in the middle panels of Fig. 4. Finally, in the fully transformed MCF-10HER2/E7 cells, HER-2/EGFR signaling regulated the expression of 480 genes that were not regulated by this signaling axis in either the MCF-10A or MCF-10HER2 cells. In the MCF-10HER2/E7 cells, 224 genes were positively regulated by HER-2/EGFR signaling, and 256 genes were negatively regulated. CAGED analysis of the expression profiles of these genes resulted in the identification of 11 clusters (Supplementary CAGED Report, pages 52-53). Graphs of the complete time series data for two genes from this category are in the bottom panels of Fig. 4. This analysis shows that HER-2 overexpression significantly altered the repertoire of genes both positively and negatively regulated by EGF in MCF-10HER2 cells compared with parental cells, and that the transition to EGF independence altered still further the repertoire of genes regulated by HER-2 signaling.

\section{Biological pathway analysis of genes regulated by EGFR/HER-2 in the isogenic cell lines}

To analyze how the changes in the repertoire of genes regulated by HER-2 in partially and fully transformed cells contribute to the expression of transformed phenotypes, we used Ingenuity Pathway Analysis to determine the canonical pathways that are statistically enriched for the differentially regulated genes. To simplify the analysis for presentation purposes, we grouped the genes from related canonical pathways into four categories, including (a) mitogen-activated protein kinase (MAPK)/ERK/phosphatidylinositol 3-kinase (PI3K)/AKT signaling, (b) integrin and cell motility signaling, (c) WNT/OCT4/Nanog/stem cell signaling, and $(d)$ stress-associated signaling including genes regulated by HIF-1a, oxidative stress, hypoxia signaling, and p38MAPK signaling. The results of the differential gene expression analysis in each of these four categories are summarized in Fig. 5. The complete gene lists for each group and for each cell line are shown in Supplementary Table $\mathrm{S} 1$.

With respect to MAPK/ERK and PI3K/AKT pathways, EGFR/HER-2 signaling positively regulated expression of seven genes and negatively regulated expression of seven genes in a similar manner in all three cell lines. Among the key genes regulated by EGFR in the three cell lines were CMYC, BCL2L1, and DUSP4. In EGF-dependent MCF-10HER2 cells, an additional seven genes were positively regulated by EGFR-HER-2 signaling, including NRAS, GSK3B, and SFN. An additional four genes were negatively regulated by EGFRHER-2 signaling in these cells, including RRAS and C-FOS. In the fully transformed, EGFindependent MCF-10HER2/E7 cells, 10 additional genes were positively regulated by HER-2/EGFR signaling, including SHC, HRAS, and PXN, and an additional 4 genes were negatively regulated, including ATF4 and ETS2. Changes in expression of genes in PI3K/ 
AKT and MAPK/ERK pathways in the cell line series are shown graphically in Supplementary Figs. S5 and S6, respectively.

A similar pattern was observed for the other canonical pathway groups that we analyzed. With respect to integrin/cell motility signaling, the number of genes positively regulated by EGFR/HER-2 signaling went from 3 genes in MCF-10A cells, to 7 genes in MCF-10HER2 cells, to 22 genes in HER-2/E7 cells. Among the genes positively regulated by HER-2/ EGFR signaling in MCF-10HER2/E7 cells were RHOC, PXN, ITGB1, and RALB, whereas ITG10 was negatively regulated in these cells. Thus, HER-2/EGFR signaling in the fully transformed cells altered the integrin repertoire of the cells and activated genes associated with motility and invasion, notably RHOC. Changes in expression of genes in these pathways in the cell line series are shown graphically in Supplementary Fig. S7.

With respect to WNT/OCT4/Nanog/stem cell pathways, overexpression of HER-2 profoundly altered gene expression patterns. EGFR signaling in MCF-10A cells did not positively regulate expression of any genes associated with these pathways and only negatively regulated two genes. However, in MCF-10AHER2 cells, EGFR/HER-2 signaling positively regulated expression of five genes and negatively regulated five genes. DKK1, a potent negative regulator of WNT signaling, was identified as a key gene whose expression was repressed by EGFR-HER-2 signaling in both HER-2-overexpressing cell lines. In the fully transformed cells, HER-2/EGFR signaling positively regulated expression of seven additional pathway genes, including FZD1, WNT10B, and SOX4. Thus, in fully transformed HER-2-overexpressing cells, several key genes in the WNT pathway were both positively and negatively regulated by HER-2, including a WNT ligand, a frizzled receptor, and a negative regulator of WNT ligand-receptor activity.

Finally, with respect to activation of stress-associated pathways, HER-2 overexpression resulted in significant alterations in gene expression. In MCF-10A cells, EGFR signaling regulated expression of four genes, one of which was negatively regulated. HER-2 overexpression resulted in positive regulation of 8 genes and negative regulation of 10 additional genes. In the fully transformed cells, HER-2/EGFR signaling resulted in positive regulation of an additional 21 genes and negative regulation of 7 additional genes. Among the key genes regulated by HER-2/EGFR signaling in the fully transformed cells are several small and large heat shock proteins, several genes that play a role in redox balance and oxidative stress, genes associated with metabolism, and genes that influence angiogenesis. Indeed, these stress-associated pathways seem to be most profoundly altered by HER-2 overexpression associated with the transition to complete growth factor independence.

\section{Altered signaling in HER-2-overexpressing cells results in an altered redox environment and activation of HIF-1 $\alpha$}

There are several predictions that can be made by examining the biological networks regulated by HER-2 in the fully transformed cells. For example, the specific downregulation of ROS-reducing enzymes such as SOD2 and GPX4 that act intracellularly and at the cell membrane suggests that the levels of ROS could be relatively high in MCF-10HER2/E7 cells. Furthermore, the upregulation of heat shock proteins suggests that these cells may be protected against the negative effects of oxidation by high levels of ROS that might otherwise cause death in a normal cell. To test these predictions, we measured ROS levels in our isogenic cell lines. Detection of ROS with a live cell assay showed that ROS levels were nearly undetectable in MCF-10A cells, were increased in MCF-10HER2 cells, and were highest in MCF-10HER2/E7 cells (Fig. 6). We then tested for evidence of increased ROS signaling by examining the function of the transcription factor HIF-1a, which is regulated by ROS in addition to hypoxia in cancer cells. Figure 7 shows the results of microarray, semiquantitative RT-PCR, and Western blot experiments, which showed that in comparison 
with MCF-10A cells, HIF-1a gene expression and protein levels were increased in MCF-10HER2 cells and increased further in MCF-10HER2/E7 cells. Next, we measured HIF-1a transcriptional activity in the isogenic cell lines by transient transfection of a HIF-1a element-luciferase reporter construct. Results in Fig. 7D showed that there was significantly more HIF-1a transcriptional activity in MCF-10HER2/E7 cells compared with MCF-10HER2 and MCF-10A cells under both normoxic and relatively hypoxic conditions. Thus, these results indicate that a key feature of the transforming function of the HER-2 oncogene, which is not found in the parental MCF-10A cells, is the generation of an altered redox environment, which secondarily results in activation of HIF-1a.

Our results show that when HER-2-overexpressing MCF-10A cells and human breast cancer cells undergo the transition to EGF independence, HER-2 becomes constitutively activated and is the dominant driver of signaling. This fundamental change in signaling coincides with the acquisition of significant malignant potential in vivo. Analysis of the gene expression changes associated with HER-2 signaling in EGF-dependent versus EGFindependent cells sheds light on how constitutively active HER-2 can mediate malignant growth potential in vivo by acquiring the capacity to regulate expression of genes associated with redox signaling, stress-associated pathways, cell motility and invasion, and WNT signaling/stem cell pathways. Thus, oncogenic HER-2 behaves in a fundamentally different way compared with the proto-oncogenic form of the protein.

\section{Discussion}

A fundamental tenet of cancer biology is that cell transformation is driven by oncogenes, the activated forms of cellular proto-oncogenes that become activated by DNA alterations involving gene amplification, gene translocation, or point mutation. It is also well accepted that activated forms of cellular proto-oncogenes are hyperactive and unregulated in their biochemical activity compared with their wild-type counterparts $(27,28)$. Nevertheless, we continue to have a poor understanding of precisely how activated oncogenes transform cells and induce phenotypes not expressed by normal cells. In the work reported here, we used a series of human mammary epithelial cell lines and human breast cancer cell lines derived by us to investigate how HER-2 is altered in its signaling properties, and how that translates into changes in gene expression when it becomes fully activated as an oncogene. The results of these experiments provide important insights into the activation of HER-2. Notably, the results point to differences in biological networks that are regulated by HER-2 as cells undergo transformation from normal, to partially transformed, to fully transformed malignant cells. Whereas it is common to consider the role of oncogenes such as HER-2 in regulation of cell proliferation and survival, the results of our systems-based analysis shows that the activated $H E R$ - 2 oncogene regulates many biological networks and pathways that contribute to the transformed phenotypes of cancer cells. Further, the data show that targeted kinase inhibitors such as gefitinib or CP724,714 have profound biological effects on cells and alter the expression of dozens of distinct gene groups. Thus, the biological properties of cells treated with such inhibitors are profoundly altered, which could be used to an advantage when considering the design of combination therapies.

Over the past several years, several investigators have examined HER family signaling receptors as a model for a systems approach to cell signaling. Wiley, Lauffenburger, and colleagues have extensively studied the molecular interactions between all four HER family members in well-defined systems, as a function of the numbers and proportions of the HER family members expressed (29-34). As a result of this work, they have developed elegant computationally based models that explain many aspects of HER family signaling in different contexts, and with regard to different phenotypes expressed by cells following ligand stimulation. Indeed, the gene expression networks that we obtained using 
MCF-10HER2 cells that are EGF dependent for growth can likely be explained by the differences in signaling potency and duration that has been elucidated by the Wiley and Lauffenburger labs. It is important to note that the studies described above, as well as our own, have focused on transcriptional effects of HER-2 signaling in different contexts. In addition, HER-2 overexpression also significantly alters the phospho-proteome in ways that influence the behavior of the transformed cells, particularly cell migration and proliferation (35).

In previous studies and in the current work, we have shown that in fully transformed cells, HER-2 can mediate the complete escape from the growth factor requirements of normal mammary epithelial cells $(6,17,19,20,36)$. Here, we have shown that in fully transformed MCF-10HER2/E7 cells and in HER-2-amplified human breast cancer cells, HER-2 is constitutively active as a kinase, is tyrosine phosphorylated to high levels, and is the dominant driver of EGFR and HER-3 tyrosine phosphorylation. This is in contrast to normal cells or to HER-2-overexpressing cells that have not completed the transition to growth factor independence, in which HER-2 phosphorylation is dependent on EGFR activation. The mechanism by which HER-2 becomes constitutively active is not understood. Our data suggest that HER-2 overexpression alone is not sufficient to induce this property because MCF-10HER2 cells express 10 -fold more $185^{\mathrm{HER}-2}$ as parental cells yet remain EGF dependent. However, all EGF-independent cells express still higher levels of the oncoprotein, ranging from 40\% higher in MCF-10HER2/E7 cells to 3-fold higher in HER-2-amplified cells (Supplementary Fig. S1). Thus, there may be a threshold of HER-2 overexpression required to mediate EGF independence. Alternatively, it is possible that HER-2 interacts with other genetic alterations, resulting in complete growth factor autonomy because MCF-10HER2/E7 cells and H16N2-HER-2 express one or more HPV-16 oncoproteins and all HER-2-amplified HBC cells have other important genetic alterations, such as p53 mutations, as well as point mutations and amplifications of other oncogenes (20). We have also performed experiments to determine if the results obtained with HER-2-overexpressing, EGF-independent cells can be explained by autocrine acting neuregulins, and we have consistently failed to find evidence for such a mechanism.

To dissect how HER-2 signaling altered downstream gene expression in our isogenic cell line series, we exploited the inhibitors described above to experimentally perturb our cell lines and analyze the gene expression changes using a bio-informatic network analysis tool. Ingenuity Pathway Analysis of genes regulated by HER-2 in fully transformed cells compared with their isogenic parental cells revealed striking differences in the biological networks regulated by HER-2 in fully transformed cells compared with growth factordependent cells or MCF-10A cells. Indeed, we found that the networks and pathways related to hypoxia, inflammation, and the heat shock response were among the most prominent distinguishing features of HER-2 as an oncogene compared with HER-2 acting as a protooncogene. Furthermore, the components of the inflammatory network predicted that the fully growth factor-independent cells would exhibit high levels of ROS and constitutive activation of HIF-1a, and these predictions were borne out experimentally. In addition, we found evidence for striking HER-2-mediated changes in WNT/stem cell signaling, integrin/ motility signaling, aryl hydrocarbon receptor signaling, fatty acid metabolism, apoptosis signaling, peroxisome proliferator-activated receptor signaling, and caveolar-mediated endocytosis. Thus, alterations in HER-2 signaling that occur as the oncogene acquires full transforming potential have a profound effect on many aspects of the biology of the transformed cells.

Much progress has been made in the development of new cancer drugs that target specific protein products of oncogenes. Clinical studies have revealed efficacy in many cases, but significant side effects have also been observed. In retrospect, these side effects should have 
been expected because targeted agents block the activity of both the oncogenic and protooncogenic activities of their targets. Indeed, the cardiotoxicity of HER-2-targeted agents helped to shed light on the role of HER family signaling in cardiomyocytes, particularly in development $(37,38)$. The analysis that we have reported here allows us to analyze how oncogenes differ from their proto-oncogenic forms biologically when they become activated and acquire full dominant transforming function. This may allow for the development of even more specifically targeted therapeutic approaches directed toward the biological networks operative in transformed cells and not in normal cells, which could increase efficacy still further, while improving the therapeutic index of new drugs even more.

\section{Supplementary Material}

Refer to Web version on PubMed Central for supplementary material.

\section{Acknowledgments}

Grant Support

All authors were supported by Dr. Ethier's Department of Defense grant DODW81XWH-08-0160, supporting National Oncogenomic and Molecular Imaging Center. The Genomics Core of the Karmanos Cancer Institute is supported by grant number P30-CA022453-29.

\section{References}

1. Lenahan C, Dennis C, Isakovich NV, Pories SE. Breast cancer: what's HER-2/neu got to do with it? Curr Surg. 2005; 62:459-464. [PubMed: 16125599]

2. Pupa SM, Tagliabue E, Menard S, Anichini A. HER-2: a biomarker at the crossroads of breast cancer immunotherapy and molecular medicine. J Cell Physiol. 2005; 205:10-18. [PubMed: 15887236]

3. Zhou BP, Li Y, Hung MC. HER-2/Neu signaling and therapeutic approaches in breast cancer. Breast Dis. 2002; 15:13-24. [PubMed: 15687642]

4. Peles E, Levy RB, Ullrich A, Yarden Y. Oncogenic forms of the neu/HER-2 tyrosine kinase are permanently coupled to phospholipase C- $\gamma$. EMBO J. 1991; 10:2077-2086. [PubMed: 1676673]

5. Chazin VR, Kaleko M, Miller AD, Slamon DJ. Transformation mediated by the human HER-2 gene independent of the epidermal growth factor receptor. Oncogene. 1992; 7:1859-1866. [PubMed: 1354348]

6. Woods Ignatoski K, Dziubinski ML, Ammerman CA, Ethier SP. Cooperative interactions of HER-2 and HPV-16 oncoproteins in the malignant transformation of human mammary epithelial cells. Neoplasia. 2005; 7:788-798. [PubMed: 16207481]

7. Diehl KM, Grewal N, Ethier SP, Woods-Ignatoski KM. p38MAPK-activated AKT in HER-2 overexpressing human breast cancer cells acts as an EGF-independent survival signal. J Surg Res. 2007; 142:162-169. [PubMed: 17612563]

8. Siegel PM, Ryan ED, Cardiff RD, Muller WJ. Elevated expression of activated forms of Neu/ ErbB-2 and ErbB-3 are involved in the induction of mammary tumors in transgenic mice: implications for human breast cancer. EMBO J. 1999; 18:2149-2164. [PubMed: 10205169]

9. Muller WJ, Ho J, Siegel PM. Oncogenic activation of Neu/ErbB-2 in a transgenic mouse model for breast cancer. Biochem Soc Symp. 1998; 63:149-157. [PubMed: 9513719]

10. Matsuda S, Akiyama T, Yamada Y, et al. Regulation of expression and transforming ability of the c-erbB-2 gene. Princess Takamatsu Symp. 1989; 20:93-101. [PubMed: 2577338]

11. Tzahar E, PinkasKramarski R, Moyer JD, et al. Bivalence of EGF-like ligands drives the ErbB signaling network. EMBO J. 1997; 16:4938-4950. [PubMed: 9305636]

12. Pinkaskramarski R, Soussan L, Waterman H, et al. Diversification of Neu differentiation factor and epidermal growth factor signaling by combinatorial receptor interactions. EMBO J. 1996; 15:2452-2467. [PubMed: 8665853] 
13. Burgess AW, Cho H-S, Eigenbrot C, et al. An open-and-shut case? Recent insights into the activation of EGF/ErbB receptors. Mol Cell. 2003; 12:541-552. [PubMed: 14527402]

14. Junttila TT, Akita RW, Parsons K, et al. Ligand-independent HER-2/HER3/PI3K complex is disrupted by trastuzumab and is effectively inhibited by the PI3K inhibitor GDC-0941. Cancer Cell. 2009; 15:429-440. [PubMed: 19411071]

15. Ethier SP, Cundiff KC. Importance of extended growth potential and growth factor independence on in vivo neoplastic potential of primary rat mammary carcinoma cells. Cancer Res. 1987; 47:5316-5322. [PubMed: 3115564]

16. Ethier SP, Chiodino C, Jones RF. Role of growth factor synthesis in the acquisition of insulin/ insulin-like growth factor I independence in rat mammary carcinoma cells. Cancer Res. 1990; 50:5351-5357. [PubMed: 1696849]

17. Ethier SP, Moorthy R, Dilts CA. Secretion of an epidermal growth factor-like growth factor by epidermal growth factor-independent rat mammary carcinoma cells. Cell Growth Diff. 1991; 2:593-602. [PubMed: 1726054]

18. Ethier SP, Langton BC, Dilts CA. Growth factor-independent proliferation of rat mammary carcinoma cells by autocrine secretion of neu-differentiation factor/heregulin and transforming growth factor-a. Mol Carcinogen. 1996; 15:134-143.

19. Ram TG, Dilts CA, Dziubinski ML, Pierce LJ, Ethier SP. Insulin-like growth factor and epidermal growth factor independence in human mammary carcinoma cells with c-erbB-2 gene amplification and progressively elevated levels of tyrosine phosphorylated erbB-2. Mol Carcinogen. 1996; 15:227-238.

20. Woods Ignatoski KM, LaPointe AJ, Radany EH, Ethier SP. ErbB-2 overexpression in human mammary epithelial cells confers growth factor independence. Endocrinology. 1999; 140:36153622. [PubMed: 10433219]

21. Woods Ignatoski KM, Livant DL, Markwart S, Grewal NK, Ethier SP. The role of PI3'kinase and its downstream signals in erbB-2-mediated transformation. Mol Cancer Res. 2003; 1:551-560. [PubMed: 12754302]

22. Fillmore CM, Kuperwasser C. Human breast cancer cell lines contain stem-like cells that selfrenew, give rise to phenotypically diverse progeny and survive chemotherapy. Breast Cancer Res. 2008; 10:R25. [PubMed: 18366788]

23. Livak KJ, Schmittgen TD. Analysis of relative gene expression data using real-time quantitative PCR and the 2(- $\Delta \Delta C(\mathrm{~T}))$ method. Methods. 2001; 25:402-408. [PubMed: 11846609]

24. Jani JP, Finn RS, Campbell M, et al. Discovery and pharmacologic characterization of CP-724,714, a selective ErbB2 tyrosine kinase inhibitor. Cancer Res. 2007; 67:9887-9893. [PubMed: 17942920]

25. Band V, Sager R. Distinctive traits of normal and tumor derived human mammary epithelial cells expressed in a medium that supports long-term growth of both cell types \#202. Proc Natl Acad Sci U S A. 1989; 86:1249-1253. [PubMed: 2919173]

26. Ramoni MF, Sebastiani P, Kohane IS. Cluster analysis of gene expression dynamics. Proc Natl Acad Sci U S A. 2002; 99:9121-9126. [PubMed: 12082179]

27. Hanahan D, Weinberg RA. The hallmarks of cancer. Cell. 2000; 100:57-70. [PubMed: 10647931]

28. Weinberg RA. The molecular basis of oncogenes and tumor suppressor genes. Ann N Y Acad Sci. 1995; 758:331-338. [PubMed: 7625701]

29. Lazzara MJ, Lauffenburger DA. Quantitative modeling perspectives on the ErbB system of cell regulatory processes. Exp Cell Res. 2009; 315:717-725. [PubMed: 19022246]

30. Joslin EJ, Opresko LK, Wells A, Wiley HS, Lauffenburger DA. EGF-receptor-mediated mammary epithelial cell migration is driven by sustained ERK signaling from autocrine stimulation. J Cell Sci. 2007; 120:3688-3699. [PubMed: 17895366]

31. Wolf-Yadlin A, Kumar N, Zhang Y, et al. Effects of HER-2 overexpression on cell signaling networks governing proliferation and migration. Mol Syst Biol. 2006; 2:54. [PubMed: 17016520]

32. Shankaran H, Wiley HS, Resat H. Modeling the effects of HER/ErbB1-3 coexpression on receptor dimerization and biological response. Biophys J. 2006; 90:3993-4009. [PubMed: 16533841]

33. Sachs K, Perez O, Pe'er D, Lauffenburger DA, Nolan GP. Causal protein-signaling networks derived from multiparameter single-cell data. Science. 2005; 308:523-529. [PubMed: 15845847] 
34. Hendriks BS, Orr G, Wells A, Wiley HS, Lauffenburger DA. Parsing ERK activation reveals quantitatively equivalent contributions from epidermal growth factor receptor and HER-2 in human mammary epithelial cells. J Biol Chem. 2005; 280:6157-6169. [PubMed: 15572377]

35. Kumar N, Wolf-Yadlin A, White FM, Lauffenburger DA. Modeling HER-2 effects on cell behavior from mass spectrometry phosphotyrosine data. PLoS Comput Biol. 2007; $3: \mathrm{e} 4$. [PubMed: 17206861]

36. Ram TG, Kokeny KE, Dilts CA, Ethier SP. Mitogenic activity of neu differentiation factor/ heregulin mimics that of epidermal growth factor and insulin-like growth factor-I in human mammary epithelial cells. J Cell Physiol. 1995; 163:589-596. [PubMed: 7775601]

37. Grazette LP, Boecker W, Matsui T, et al. Inhibition of ErbB2 causes mitochondrial dysfunction in cardiomyocytes: implications for herceptin-induced cardiomyopathy. J Am Coll Cardiol. 2004; 44:2231-2238. [PubMed: 15582322]

38. Negro A, Brar BK, Lee KF. Essential roles of Her2/erbB2 in cardiac development and function. Recent Prog Horm Res. 2004; 59:1-12. [PubMed: 14749494] 
A
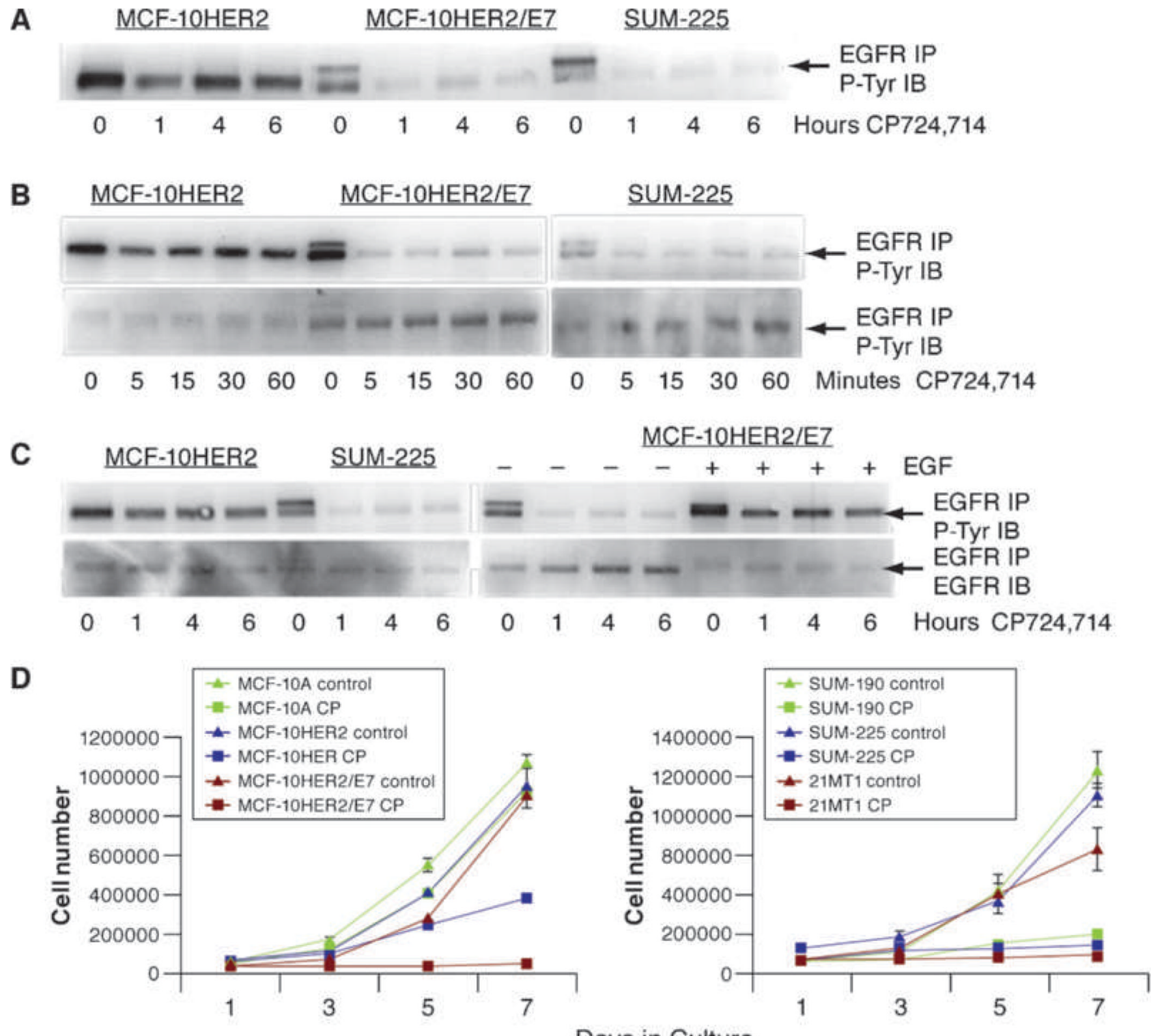

Days in Culture

Figure 1.

The transition to EGF independence results in the acquisition of dominant signaling activity by HER-2. A, MCF-10AHER2, MCF-10HER2/E7, or SUM-225 cells were exposed to CP724,714 for 1 to $6 \mathrm{~h}$, followed by EGFR immunoprecipitation (IP) and phosphotyrosine Western blotting (P-Tyr IB). CP724,714 had no effect on EGFR tyrosine phosphorylation in MCF-10HER2 cells. In MCF-10HER2/E7 and SUM-225 cells, EGFR immunoprecipitation resulted in coimmunoprecipitation of tyrosine-phosphorylated HER-2, both of which were inhibited by exposure to CP724,714. B, the identical experiment was performed as described in A, but with a 5-min exposure to drug, indicating that signaling inhibition occurs for both receptors quickly after drug exposure. $\mathrm{C}$, repeat of the experiment shown in A, but with the addition of another group consisting of MCF-10HER2/E7 cells that had been exposed to EGF overnight. The results show that when ligand activates EGFR, its tyrosine phosphorylation is not blocked by the HER-2 kinase inhibitor. D, EGF-independent, 
HER-2-transformed cells are completely dependent on HER-2 signaling for proliferation. The isogenic MCF-10A cell line series (left) and a panel of human breast cancer cell lines with HER-2 amplification (right) were cultured with $1 \mu \mathrm{mol} / \mathrm{L}$ CP724,714 (CP) for 7 to $9 \mathrm{~d}$, and cell counts were determined every other day during the course of the experiment. 


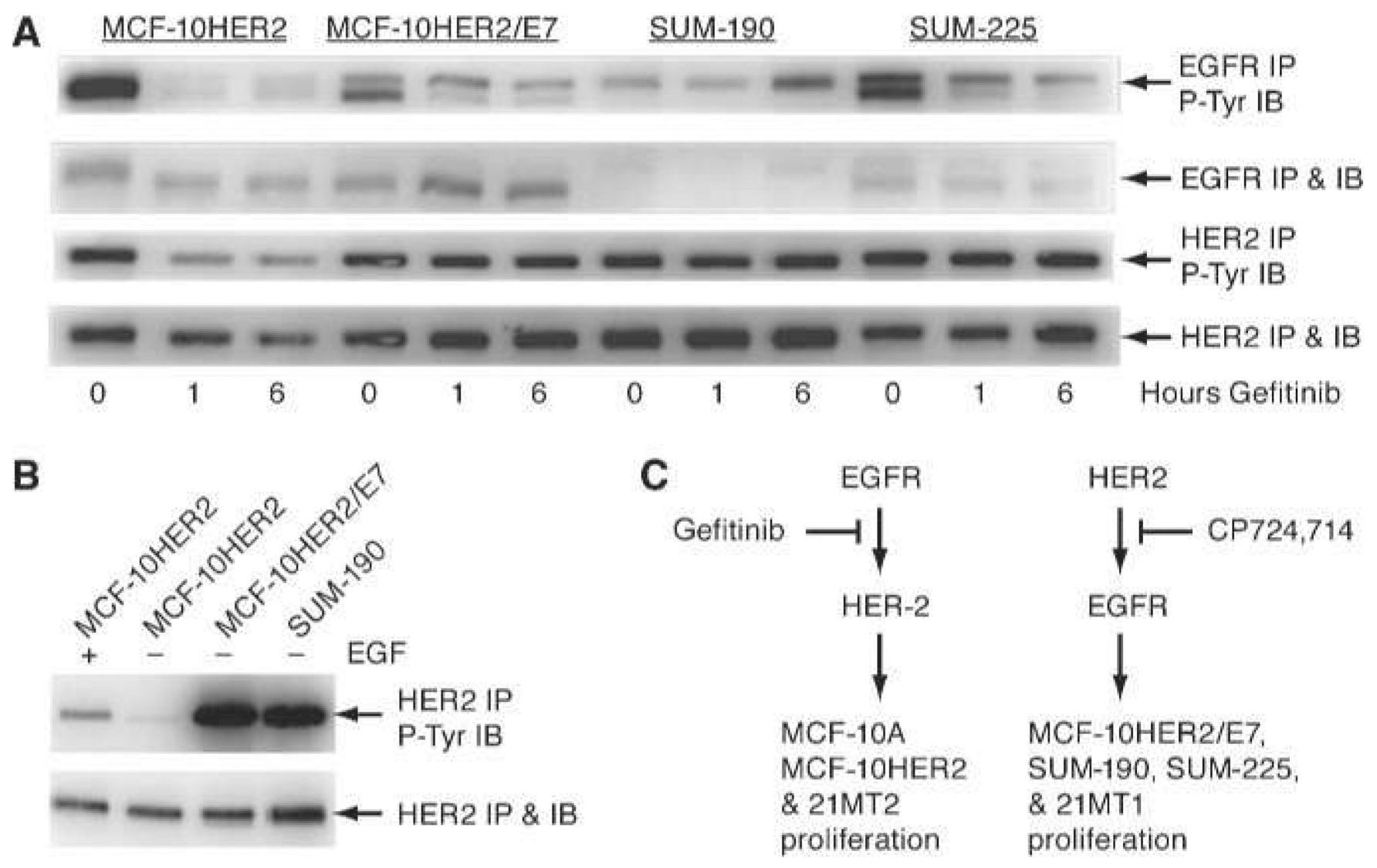

Figure 2.

HER-2 tyrosine phosphorylation is dependent on EGFR in the EGF-dependent

MCF-10HER2 cells, but independent of EGFR in the EGF-independent cell lines. A,

MCF-10HER2, MCF-10AHER2/E7, SUM-190, and SUM-225 cells were cultured in the presence of $0.5 \mu \mathrm{mol} / \mathrm{L}$ gefitinib for 1 to $6 \mathrm{~h}$ followed by either EGFR or HER-2

immunoprecipitation and phosphotyrosine Western blotting. Results show that gefitinib effectively blocked EGFR in all cell lines (note the lower band of the doublet in the EGFindependent cells), but only blocked HER-2 tyrosine phosphorylation in the EGF-dependent MCF-10HER2 cells. Gefitinib had no effect on HER-2 tyrosine phosphorylation in any of the EGF-independent cell lines (upper band of the doublet in the EGFR IP and in the HER-2 IP lanes). B, effect of EGF withdrawal on HER-2 tyrosine phosphorylation in MCF-10AHER2 cells, and the steady-state levels of HER-2 tyrosine phosphorylation in growth factor-independent MCF-10HER2/E7 cells and SUM-190 cells, which are grown routinely in EGF-free medium. C, the current signaling model based on our data, which indicates that in EGF-dependent but HER-2-overexpressing cells, EGFR is the driver of signaling and mediates its own and HER-2 tyrosine phosphorylation. In cells that have undergone the transition to growth factor independence, HER-2 is the dominant driver of signaling and mediates its own and EGFR tyrosine phosphorylation. 


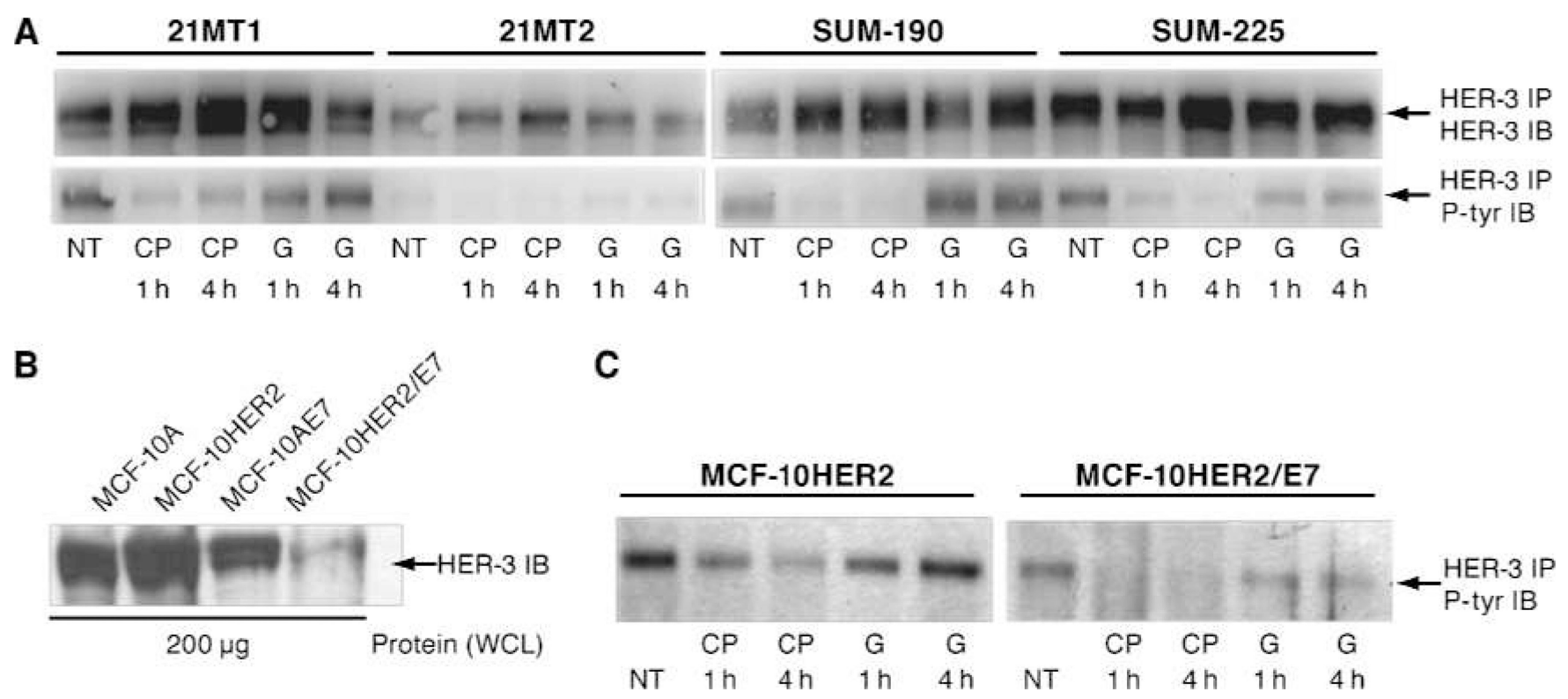

Figure 3.

HER-2 is the driver of HER-3 tyrosine phosphorylation in EGF-independent cells. Four human breast cancer cell lines with HER-2 amplification (A) and the isogenic MCF-10A cell line series (B and C) were treated with either CP724,714 or gefitinib for 1 or $4 \mathrm{~h}$ followed by HER-3 immunoprecipitation and HER-3 phosphotyrosine Western blotting. Note that 21MT2 cells, despite having a HER-2 amplification, are EGF-dependent for growth. The results show that HER-2 kinase activity is responsible for HER-3 tyrosine phosphorylation in all EGF-independent cells. 

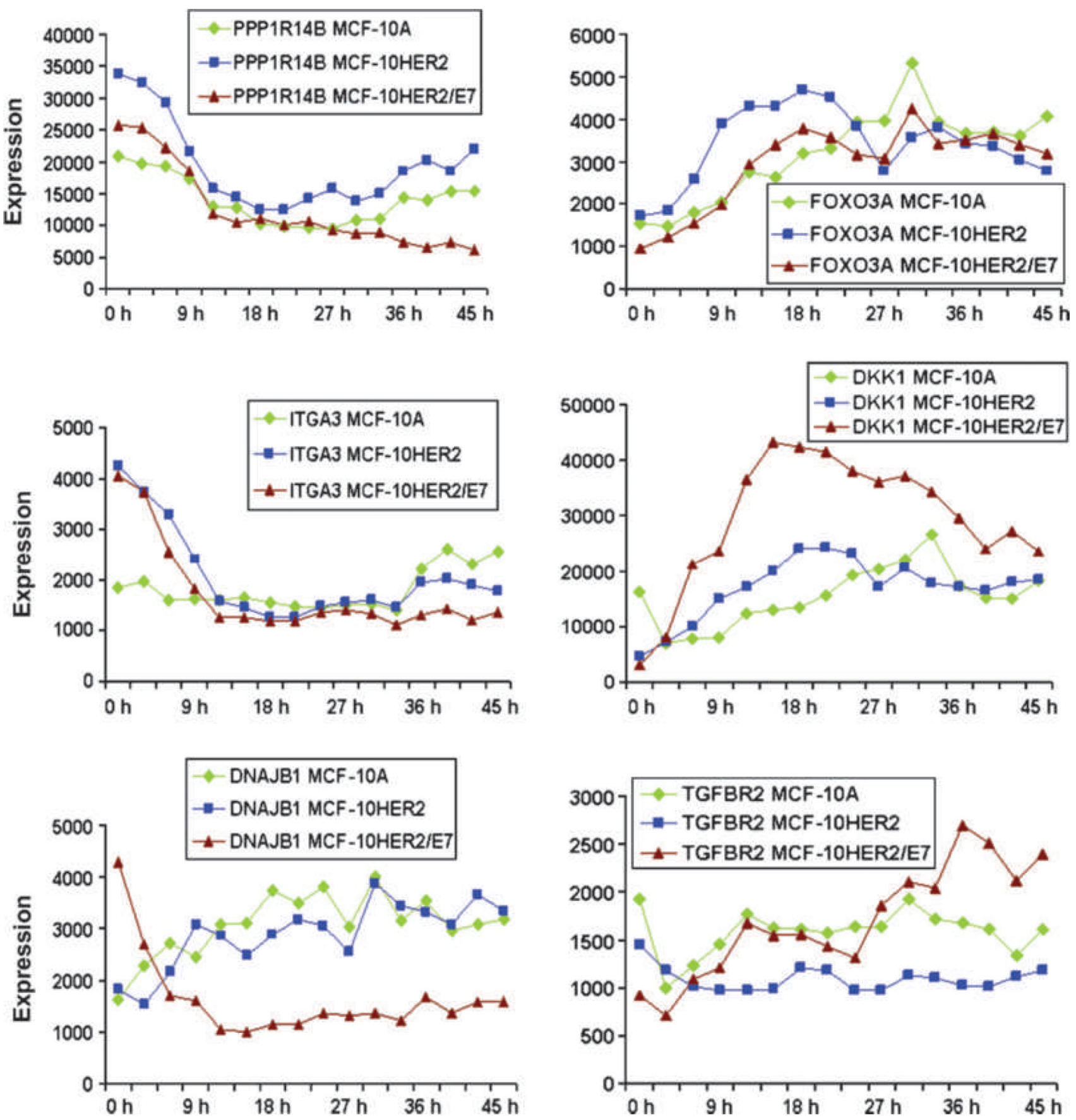

Figure 4.

Regulation of gene expression by EGFR/HER-2 signaling in the MCF-10A isogenic cell line series. Cells were cultured in the presence of either gefitinib or CP724,714 and harvested every $3 \mathrm{~h}$ for $45 \mathrm{~h}$ for RNA isolation and whole-genome expression profiling. The top two panels show examples of genes that are either positively (left) or negatively (right) regulated by EGFR/HER-2 signaling in all three cell lines. The middle two panels show examples of genes regulated by EGFR/HER-2 signaling in both HER-2-overexpressing cell lines but not in MCF-10A cells. The bottom two panels show examples of genes regulated by HER-2/ EGFR signaling in MCF-10HER2/E7 cells but not by this signaling axis in the other two cell lines. 
PI3K/AKT/ERK/MAPK

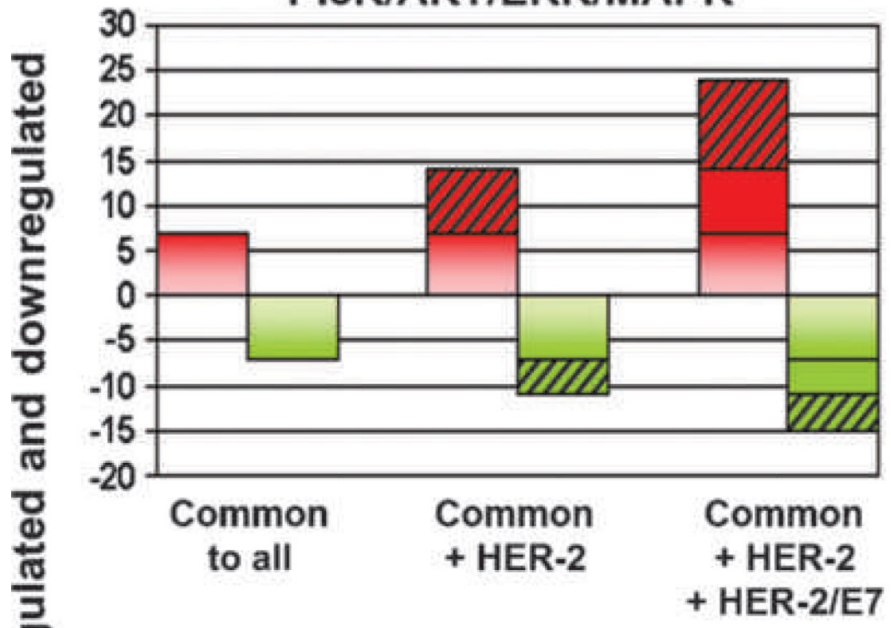

HIF-1 $\alpha /$ Oxidative stess

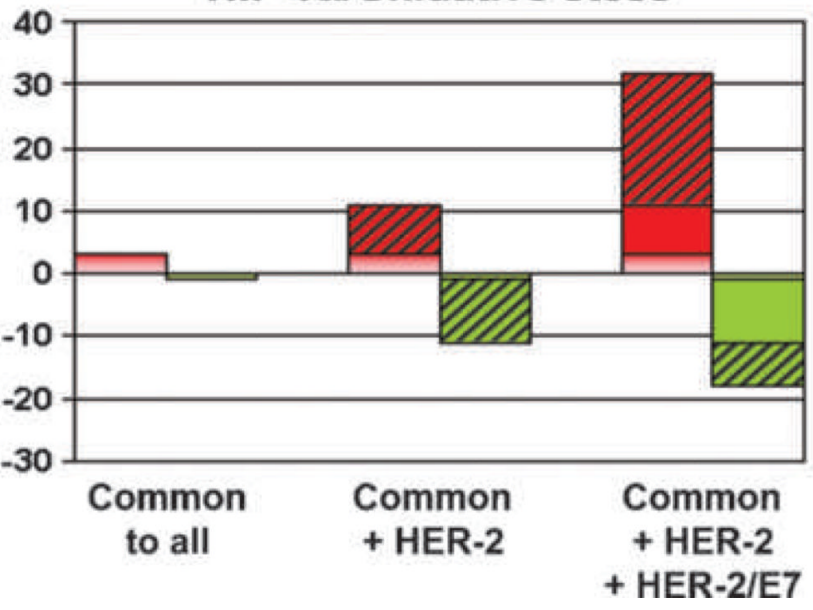

Integrin/cell motility

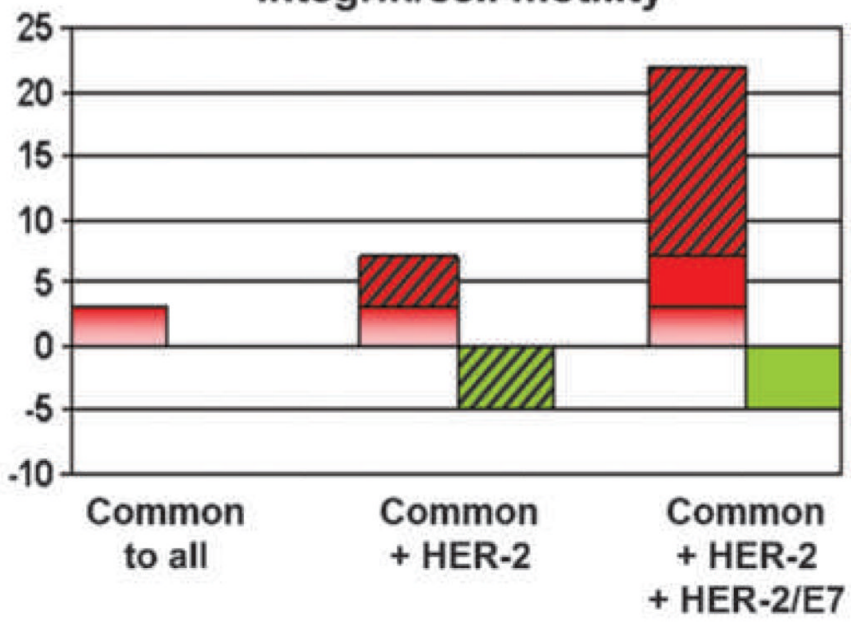

WNT/stem cell

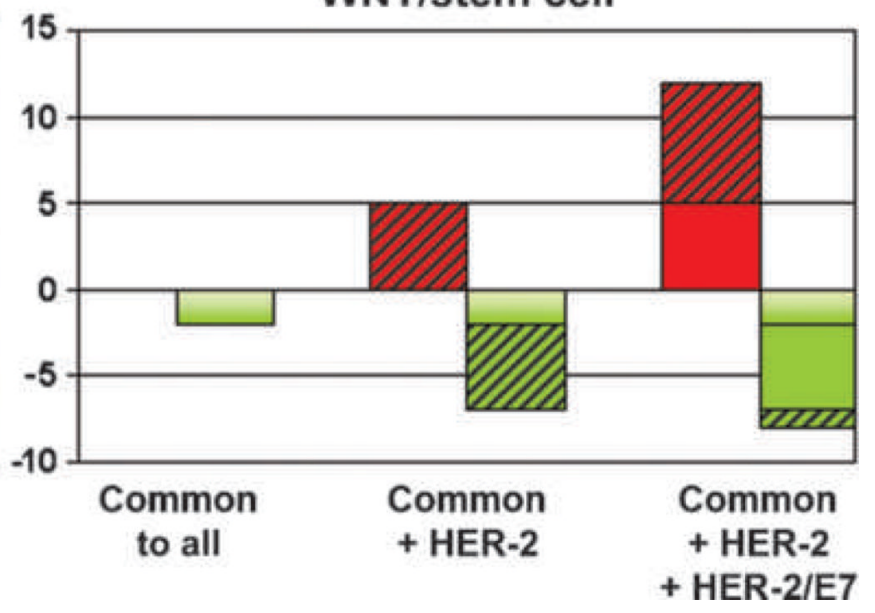

Figure 5.

The progressive acquisition of HER-2-mediated transforming activity results in dramatic changes in downstream gene regulation affecting many different biological processes. Ingenuity Pathway Analysis was performed on the genes regulated by EGFR/HER-2 signaling in the isogenic cell line series and grouped into the four biological categories based on the canonical pathways indicated in the figure. The set of bars on the far left indicates the number of common genes in each canonical pathway set that are regulated by EGFR/HER-2 signaling in all three cell lines. The middle set of bars indicates the number of common genes plus the number of genes in each canonical pathway set regulated by EGFR/ HER-2 signaling in both HER-2-overexpressing lines, but not in MCF-10A cells. The set of bars on the right indicates the number of genes regulated in all three lines, plus the genes regulated in both HER-2-overexpressing lines, plus the genes regulated by HER-2/EGFR signaling in MCF-10HER2/E7 cells, but not the other two cell lines. Red bars show the number of genes positively regulated by signaling, and green bars indicate the number of genes negatively regulated by signaling. 


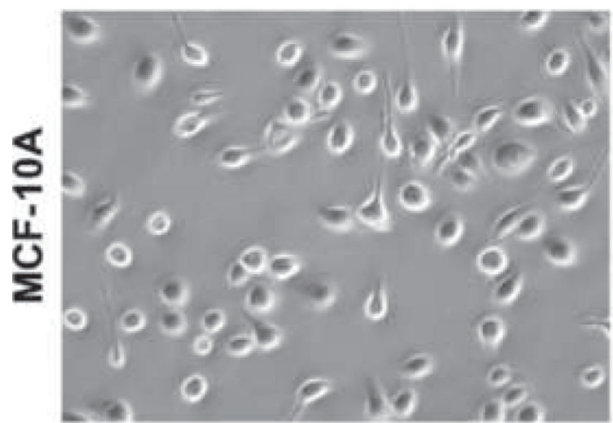

ROS staining
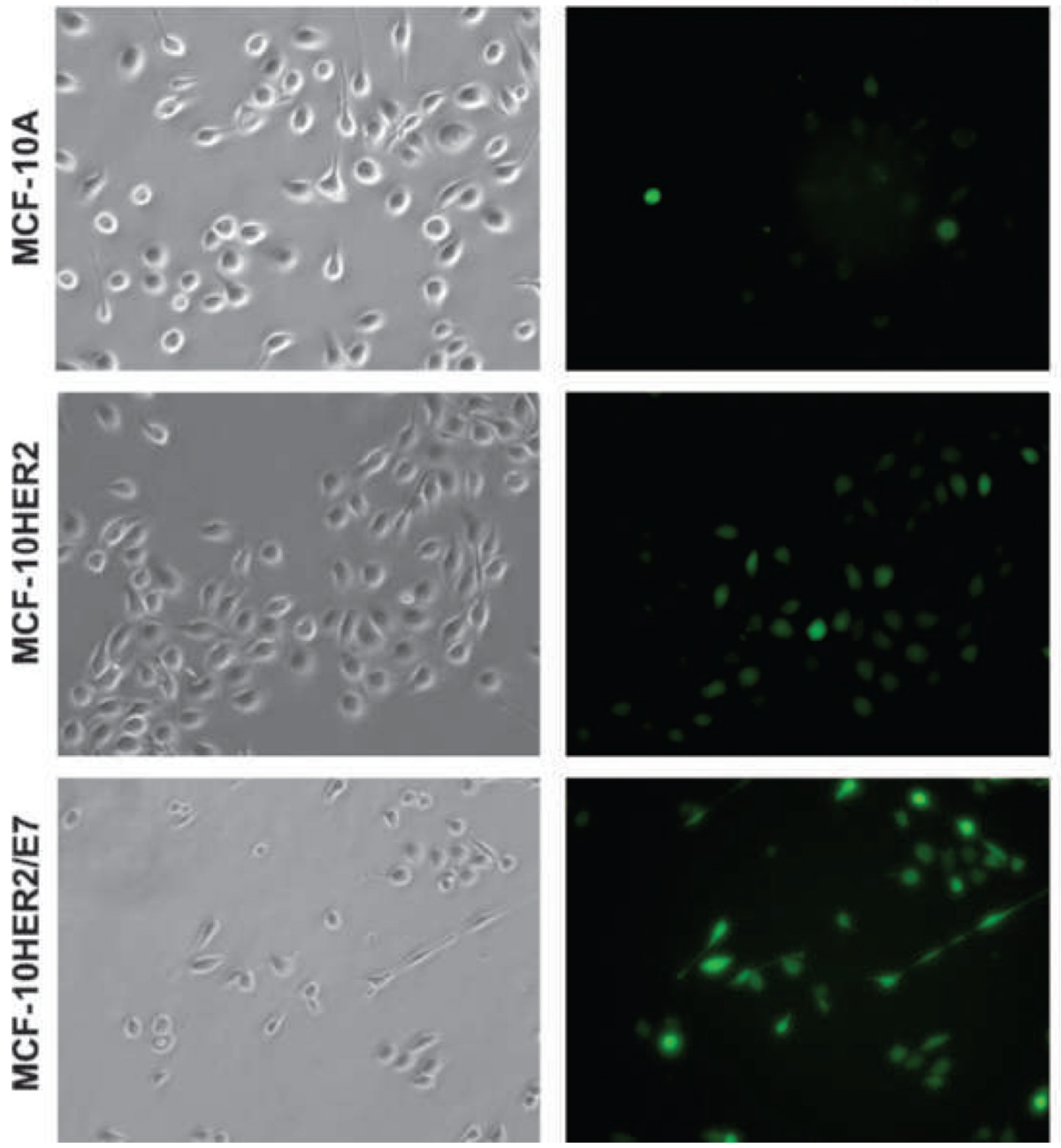
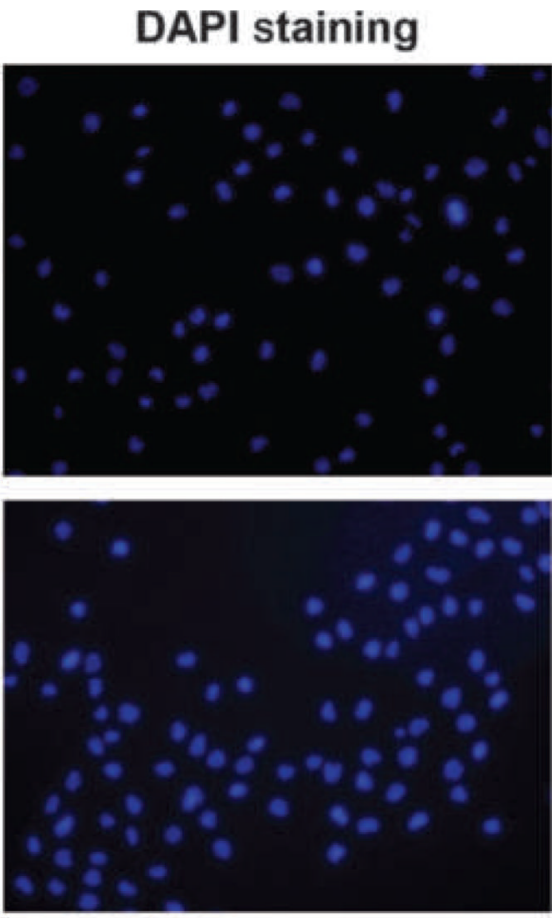

Figure 6.

Determination of ROS in the MCF-10 isogenic cell lines by incubation with 5,6-carboxy-2', $7^{\prime}$-dichlorodihydrofluorescein diacetate, a fluorogenic marker for ROS in live cells. Left, a bright-field image of cells in culture. Middle, in the same field, the oxidized fluorescein compound emits green fluorescence observed by fluorescence microscopy (excitation, 495 $\mathrm{nm}$; emission, $529 \mathrm{~nm}$ ). Right, fluorescence microscopy also detected blue-fluorescing nuclei stained with Hoechst 3342 (excitation, $350 \mathrm{~nm}$; emission, $461 \mathrm{~nm}$ ). 

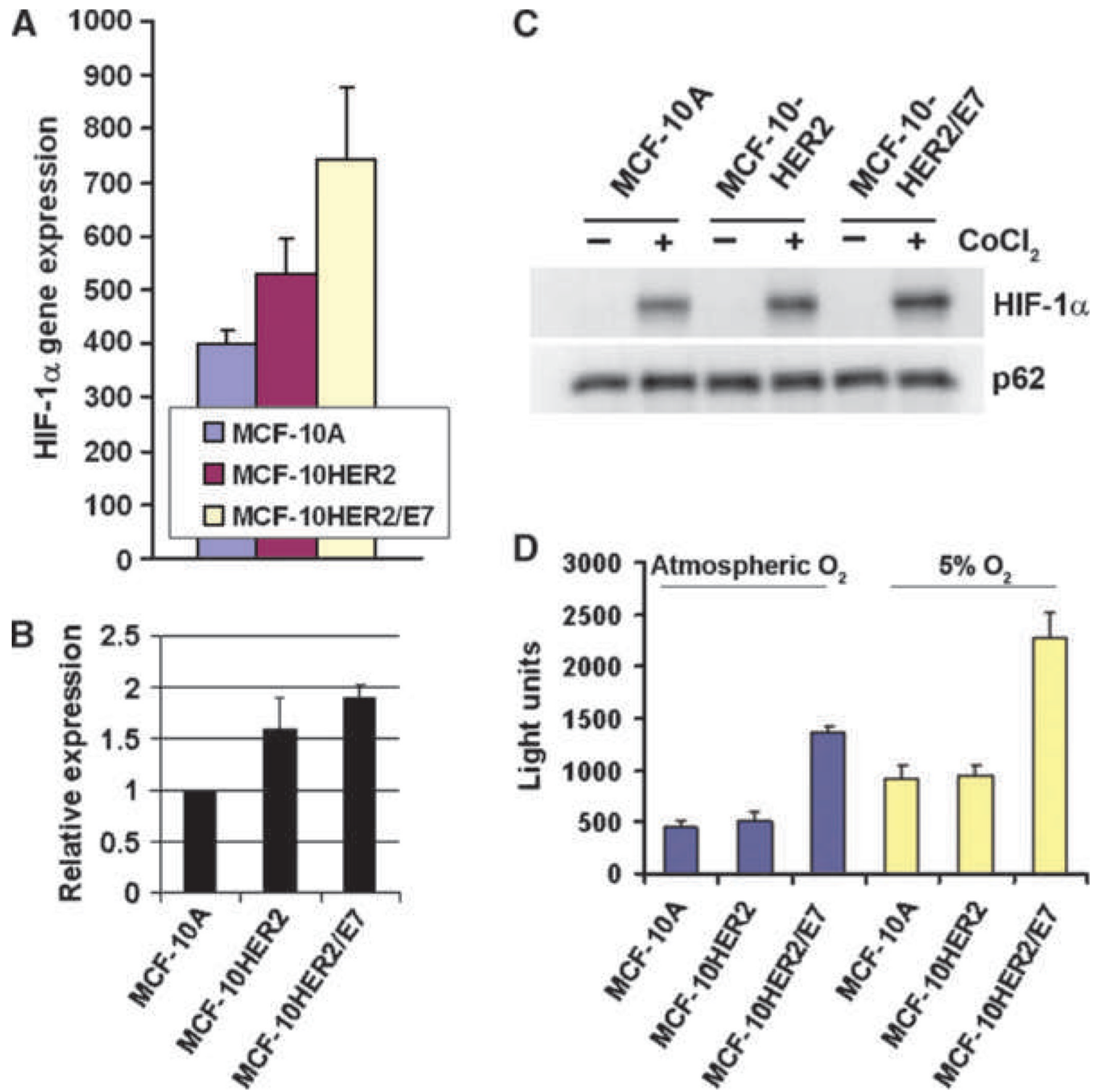

Figure 7.

HIF-1a activation in isogenic MCF-10A cell line series. A, levels of HIF-1a gene expression detected by Illumina microarray analyses (three arrays). Bars, SE. mRNA and protein levels were confirmed in these cell lines by semiquantitative RT-PCR (B) and Western blot (C). D, the MCF-10A cell line and HER-2-overexpressing MCF-10HER2 and MCF-10HER2/E7 cell lines were transiently transfected with a luciferase reporter construct driven by tandem repeats of the canonical HIF-1a response element. Transiently transfected cells were incubated either in atmospheric oxygen and $10 \% \mathrm{CO}_{2}$ or in a $5 \% \mathrm{O}_{2}$ and $10 \%$ $\mathrm{CO}_{2}$ controlled incubator. Luciferase activity, light units, was measured at $72 \mathrm{~h}$ after transfection and normalized to the amount of protein in each assay. HIF-1a reporter activity 
was induced by relative hypoxic conditions, indicating specificity of the reporter for activated HIF-1a. In both conditions, HIF-1a reporter activity was higher in the MCF-10HER2/E7 cell line compared with MCF-10A and MCF-10HER2 cells. The experiment in its entirety was repeated in triplicate. Columns, mean of assay triplicates; bars, SD. 